\title{
EFFECTS OF DISPLAY LOCATION WITHIN SIMULATED DRIVING ENVIRONMENTS
}

\author{
Matthew C. Crisler, Johnell O. Brooks, Kelly Riggins, \\ Brandon Garris, Jessica Tyler, and Sam Dahl \\ Clemson University \\ Clemson, SC, USA \\ Email: jobrook@clemson.edu
}

\begin{abstract}
Summary: Driving simulators offer researchers experimental control while minimizing safety issues and reducing costs relative to on-road and test track experimental procedures. However, with the control of the visual environment that simulators allow, it can be tempting to develop experimental protocols that utilize displays within the visual environment of the simulator. Such displays have the potential to differentially affect driving performance based on their location within the driving environment. A simulator experiment was conducted in order to assess the effects of having drivers fixate a display at nine different locations on the center channel of a DriveSafety driving simulator. In general, driving performance was best when the display was in the middle of the screen. Both horizontal and vertical deviations from the center of the screen resulted in increased lane position variability, and drivers tended to drive closer to the opposite lane boundary toward which they were fixating when a display was located to the left or right of the center. In addition, response times to a task presented in the display were faster when the display was located toward the center of the screen.
\end{abstract}

\section{INTRODUCTION}

Much driving research is conducted in driving simulators which offer experimenters a large degree of experimental control while minimizing safety issues and expenses associated with onroad and test-track procedures. These simulators consist of computer-controlled visual environments that are directly controllable by the experimenter, thereby allowing the experimenter to display all manner of visual and auditory stimuli in ways that would be impossible or extremely expensive and time consuming in a real-world environment. Unfortunately, although this ability is useful in many circumstances, it is possible that taking advantage of this ability may affect driving performance in an unintended manner. Research designed to assess the effectiveness of different methods of displaying information to drivers (Summala, Lamble, \& Laakso, 1998; Summala, Nieminen, \& Punto, 1996; Tsimhoni, Watanabe, Green, \& Friedman, 2000) suggests that driving performance may be affected differentially by placing displays at different locations within a vehicle and forcing drivers to use peripheral vision to maintain lane position.

In a study by Summalla Nieminen \& Punto (1996), drivers were asked to fixate on three different locations ranging from above the dashboard to the center console. The results of this study showed that lane keeping performance tends to decline with increasing display eccentricity (measured from sight line to vanishing point on horizon); however, the decreased lane keeping 
performance was more pronounced for novice drivers. It was hypothesized that this may be due to the fact that the novice drivers are more likely to use foveal vision to maintain lane position than the experienced drivers. Crisler et al. (2008), revealed a potentially related phenomenon while investigating distracted driving. In this study, participants were asked to speak a word out loud that began with a letter presented on the screen of a DriveSafety driving simulator. The letters were presented in the middle of the simulator's display screen and approximately at the level of the horizon. Surprisingly, even though drivers were expected to be distracted by the task, lane keeping performance was better than in a no-task control condition. It is possible that, because drivers were forced to fixate at the horizon while playing the game, they made effective use of peripheral rather than foveal vision to maintain lane position. In other words, the forced fixation near the horizon required participants to fulfill the first of the Smith System's five keys to safe driving (i.e., aim high in steering) (Johnson, Crabb, Opfer, Thiel, \& Mottola, 2007).

Summalla Lamble and Laakso (1998) suggest that displaying information within the vehicle has similar detrimental effects on perception of brake lights; however, a moderating effect of driving experience was not observed.

Additionally, driver training research using eye tracking methodologies has identified driving conditions that produce changes in driving performance and visual scanning (Chapman, Underwood, \& Roberts, 2002). Chapman, Underwood, and Roberts suggest that "regular scanning of... the visual field may be necessary to ensure that the driver rapidly becomes aware of changes in the behavior of other traffic participants" (Chapman, Underwood, \& Roberts, 2002 , p. 159). For instance, the use of certain information in simulator displays may limit drivers' ability to scan the environment. Similar research shows scanning is reduced in high cognitive load environments such as those in some driving simulator experiments (Chapman \& Underwood, 1998). Overall, these studies suggest that when designing driving studies, it is important to account for the effects displaying information at different locations within the environment may have on driving performance. This experiment was designed to assess how simulator displays that force drivers to fixate a certain location may affect driving performance variables such as lane position and lane keeping performance. It was expected that driving performance would be hindered when drivers' were forced to fixate at displays located near the bottom of the simulator display and to the sides of the simulator display.

\section{METHODS}

\section{Participants}

32 undergraduate psychology students with no self-reported visual pathologies or history or motion sickness completed the study. From this group, data were used from 27 licensed drivers (16 male) with ages ranging from 18 to $23(\mathrm{M}=18.67)$ to ensure proper counterbalancing.

\section{Apparatus}

Participants drove a fixed-base driving simulator (DriveSafety DS-600C) consisting of five projected visual channels ( $300^{\circ}$ field of view) and three LCD panels simulating the views of two 
side and one rear-view mirror. Participants sat in the body of a Ford Focus sedan and interacted with the simulator using the steering wheel, pedals, and speedometer.

The driving scenario consisted of a curvy two-lane rural road. On the forward visual channel, the simulator was programmed to display either the speed of the driver's vehicle or an arrow pointing left or right at one of nine different locations. Throughout most of the experiment the speed display was visible, but every five seconds, it was switched to a left or right arrow. Participants responded to the arrows by depressing one of the two buttons on the back left and right of the steering wheel corresponding to the direction of the arrow presented on the screen. Once a correct response was received, the arrow was removed and replaced by the speed display. Each location was fixated for 60 seconds (approximately 11 arrow presentations).

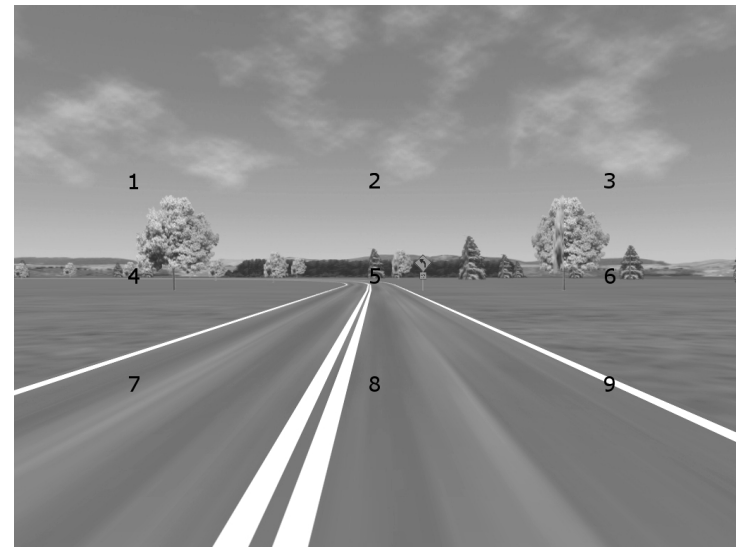

Figure 1. Simulator front channel with 9 locations marked

The simulator collected data at $10 \mathrm{~Hz}$ including: lane position, lane position variability, speed, speed variability, percentage of time spent within lane, and average response time to the arrow display. Accuracy was not measured because pilot testing showed high accuracy across conditions and participants were required to give a correct response in order to continue.

\section{Experimental Design}

A 3 X 3 repeated measures design was used with three levels of vertical eccentricity and three levels of horizontal eccentricity. The order in which the locations were presented was counterbalanced using a balanced latin squares design.

\section{Procedure}

After adjusting the seat and controls for comfort, participants completed three practice sessions on a straight road and two curvy roads. During the first two practice sessions, a lane deviation indicator appeared on the front visual channel when the participant was not completely within the appropriate lane. The third practice session provided a baseline driving performance measure and ensured that all participants could effectively drive the simulated vehicle prior to the experimental trials. 
After the practice sessions, participants completed experimental sessions with two tasks designed to force them to fixate at the location of the arrow/speed display. The arrows task required participants to fixate on the arrow/speed display and respond to arrows displayed every 5 seconds by depressing the left or right button on the back of the steering wheel. The speed maintenance task required drivers to remain within a speed range of 53-57 MPH throughout the experimental session. Participants were given a monetary incentive (a percentage of ten dollars) corresponding to the percentage of time they remained within the speed range.

\section{Data Analysis}

A series of 3 X 3 (vertical X horizontal eccentricity) repeated measure ANOVAs were used to identify differences in dependent measures. LSD post-hoc follow up tests were completed when significant differences were identified. As appropriate Greenhouse-Geisser degrees of freedom adjustments were used to adjust for violations of sphericity.

\section{RESULTS}

\section{Response Time}

An ANOVA on the response time data showed significantly different response times for both horizontal, $F(1.5,39.5)=14.5, p<0.001$, and vertical eccentricity, $F(1.7,43.4)=3.89, p=0.035$. Post hoc comparisons for the horizontal eccentricity variable revealed significantly faster response times in the middle locations as compared to the outer locations (left and right), $p<0.001$. Post hoc comparisons for the vertical eccentricity variable revealed significantly faster response times for the middle and bottom locations relative to the top locations, $p=0.031$ and $p=0.047$ respectively. There was no significant difference between the bottom and middle locations, $p=0.835$. Table 1 shows mean response times at each of the nine locations.

Table 1. Response times (SD) in ms at each location

\begin{tabular}{llll}
\hline Location & Left & Middle & Right \\
\hline Top & $89.1(19.1)$ & $78.4(16.8)$ & $87.8(18.3)$ \\
Middle & $82.9(13.5)$ & $77.0(17.6)$ & $83.6(17.3)$ \\
Bottom & $85.8(17.5)$ & $75.5(18.4)$ & $83.0(18.9)$ \\
\hline
\end{tabular}

\section{Lane Position}

An ANOVA on the lane position data revealed a significant main effect of horizontal eccentricity, $F(1.4,36.2)=13.918, p<0.001$. All other main and interaction effects were nonsignificant $(p>0.05)$. Post hoc comparisons revealed that drivers drove closer to the left lane boundary when fixating at a location to the right as compared to when looking to the left or center and vice versa $(p=0.001)$. There was also a significant difference in lane position when drivers fixated on the right side targets relative to the center targets. Again, fixating to the right tended to cause the drivers to drive closer to the left lane boundary, $p<0.001$. As shown in Table 2 , this effect is most pronounced when drivers are looking down at the targets located at or near 
the lane boundaries, and the deviation from the center of the lane is larger when looking to the right as compared to looking left. This interaction effect was nonsignificant.

Table 2. Mean lane position (SD) in meters from center

\begin{tabular}{llll}
\hline Location & Left & Middle & Right \\
\hline Top & $0.01(0.15)$ & $0.03(0.10)$ & $-0.02(0.11)$ \\
Middle & $0.02(0.12)$ & $0.00(0.11)$ & $-0.06(0.10)$ \\
Bottom & $0.03(0.12)$ & $-0.01(0.11)$ & $-0.07(0.09)$ \\
\hline
\end{tabular}

\section{Lane Position Variability}

An ANOVA on the lane position variability measure (SD of lane position) revealed significant main effects of both horizontal and vertical eccentricity $(F(2,52)=8.1, p=0.001$ and $F(1.6$, $41.9)=7.1, p=0.004$ respectively). The interaction effect of horizontal and vertical eccentricity was nonsignificant, $p=0.294$. Post hoc comparisons revealed that lane position variability was significantly higher when fixating to the left or right $(\mathrm{M}=0.233$ and 0.229 respectively compared to $\mathrm{M}=0.208$ for the middle displays), $p<0.001$ for left compared to middle and $p=0.010$ for right compared to middle. Post hoc comparisons between the three levels of vertical eccentricity showed a similar pattern where the variability was lower in the middle locations $(\mathrm{M}=0.210)$ as compared to the top and bottom locations $(\mathrm{M}=0.227$ and $\mathrm{M}=0.233$ respectively), $p=0.002$ and $p=0.001$. Table 3 shows the lane position variability at each of the nine display locations.

Table 3. Mean lane position variability (SD) in meters

\begin{tabular}{llll}
\hline Location & Left & Middle & Right \\
\hline Top & $0.23(0.09)$ & $0.22(0.06)$ & $0.24(0.09)$ \\
Middle & $0.23(0.08)$ & $0.18(0.09)$ & $0.22(0.08)$ \\
Bottom & $0.24(0.10)$ & $0.22(0.08)$ & $0.23(0.13)$ \\
\hline
\end{tabular}

\section{Percentage of Time With Any Portion of Vehicle Outside Lane}

An ANOVA on the data representing the percentage of time that participants spent outside their lane revealed significant main effects of both horizontal and vertical eccentricity $(F(2,52)=6.9$, $p=0.002$ and $F(2,52)=4.1, p=0.022$ respectively). The interaction of horizontal and vertical eccentricity was not significant, $p=0.538$. Post hoc comparisons revealed a similar pattern to the lane position variability measure where the two outer display locations resulted in significantly more time spent outside the lane as compared to the middle location, $p=0.002$ and $p=0.003$ for left compared to middle and middle compared to right respectively. The left and right locations were not significantly different from each other, $p>0.05$. Post hoc comparisons for vertical eccentricity revealed that the bottom locations resulted in significantly more time spent outside the lane as compared to the middle location, $p=0.010$. The middle and high locations did not result in a significant difference in time spent outside the lane, $p>0.05$. Table 4 shows the percentage of time spent outside the lane for each of the nine display locations. 
Table 4. Mean percentage of time spent out of lane

\begin{tabular}{lccc}
\hline Location & Left & Middle & Right \\
\hline Top & $4.0 \%(0.06)$ & $1.8 \%(0.02)$ & $3.8 \%(0.06)$ \\
Middle & $3.6 \%(0.05)$ & $1.8 \%(0.04)$ & $2.7 \%(0.04)$ \\
Bottom & $4.2 \%(0.07)$ & $2.6 \%(0.05)$ & $5.0 \%(0.07)$ \\
\hline
\end{tabular}

\section{Speed and Speed Variability}

An ANOVA on the speed and speed variability (SD of speed) data revealed no significant differences between conditions, $p>0.05$. The average speed across all locations was 55.23 MPH. The average speed variability (SD of speed) was $0.66 \mathrm{MPH}$ across all locations.

\section{DISCUSSION}

This study was designed to address the effects of displays within simulated driving environments on driving performance variables. As expected, the use of information displays at different locations within the simulated driving environment led to notable differences in driving behavior and response time to the display. It is important to note that although there were differences in driving performance with different display locations, most were fairly small. Therefore, although it is desirable to limit the use of such displays, research using displays within the simulated driving environment is not necessarily invalid. When designing studies in these environments, however, researchers must take care to limit the effects of the displays they use on the variables that they are measuring.

As noted previously, response time to arrow displays was faster when the display was closer to the center of the screen. Although the response time measure suggests that outer locations are less effective than central locations, participants' speeds were not affected by the display locations, indicating that they were reasonably capable of using the outer displays to monitor their speed.

As for changes in mean lane position, the tendency for drivers to drive further from the lane boundary that they were looking fits with the Smith System which encourages drivers to "aim high in steering" (Johnson, Crabb, Opfer, Thiel, \& Mottola, 2007, p. 64). That is, by aiming high, drivers can use peripheral vision to maintain lane position while scanning the environment for relevant events. This prevents weaving back and forth within the lane when looking down and alternating between the left and right lane boundary. Although this effect is not extremely large (a lateral shift of ten centimeters), it is important that researchers understand the effects of manipulating display location when designing experiments.

In addition to the lane position changes that resulted from changes in display location, the lane position variability and percentage of time spent outside the lane followed a similar pattern where the outer display locations (left, right, top, and bottom) resulted in higher lane position variability and increased time outside the boundaries of the lane. Although these changes are also relatively small (a six centimeter change in lane position variability and $3.2 \%$ change in time 
outside the lane), they represent differences that could make significant changes to the results of an experiment.

\section{CONCLUSION}

It is important when designing research studies as well as training or assessment protocols in driving simulators to ensure that the displays used do not affect the outcome of the simulation. Although the changes in driving performance identified by this study are relatively small, they could result in meaningful changes in the results of simulator studies. In the future, other potential issues with simulator displays, such as visual scanning changes, need to be addressed and defined so that researchers may more effectively design studies within simulated environments.

\section{REFERENCES}

Chapman, P. R., \& Underwood, G. (1998). Visual Search of Driving Situations: Danger and Experience. Perception, 27, 951-964.

Chapman, P., Underwood, G., \& Roberts, K. (2002). Visual Search Patternsin Trained and Untrained Novice Drivers. Transportation Research Part F , 5, 157-167.

Crisler, M. C., Brooks, J. O., Ogle, J. H., Guirl, C., Alluri, P., \& Dixon, K. (2008). Effect of Wireless Communication and Entertainment Devices on Simulated Driving Performance. TRB 87'th Annual Meeting Compendium of Papers DVD. Washington: Transportation Research Board.

Johnson, M. L., Crabb, O., Opfer, A. A., Thiel, R. R., \& Mottola, F. R. (2007). Drive Right. Boston, MA: Pearson Prentice Hall.

Summala, H., Lamble, D., \& Laakso, M. (1998). Driving Experience and Perception of The Lead Car's Braking When Looking at In-Car Targets. Accident Analysis and Prevention, 30 (4), 401-407.

Summala, H., Nieminen, T., \& Punto, M. (1996). Maintaining Lane Position with Peripheral Vision During In-Vehicle Tasks. Human Factors , 38 (3), 442-451.

Tsimhoni, O., Watanabe, H., Green, P., \& Friedman, D. (2000). Display of Short Text Messages on Automotive HUDs: Effects of Driving Workload and Message location. University Of Michigan. Ann Arbor: UMTRI. 